\title{
Configuration Matrix Design of Over-Actuated Marine Systems
}

\author{
Huu-Tho Dang \\ LIRMM institute \\ Montpellier University \\ Montpellier, France \\ tho.dang-huu@lirmm.fr \\ Lionel Lapierre \\ LIRMM institute \\ Montpellier University \\ Montpellier, France \\ lapierre@lirmm.fr
}

\author{
Rene Zapata \\ LIRMM institute \\ Montpellier Univeristy \\ Montpellier, France \\ zapata@lirmm.fr
}

\author{
Pascal Lepinay \\ LIRMM institute \\ Montpellier Univeristy \\ Montpellier, France \\ lepinay@lirmm.fr
}

\author{
Benoit Ropars \\ LIRMM institute \\ Montpellier Univeristy \\ Montpellier, France \\ benoit.ropars@lirmm.fr
}

\begin{abstract}
This paper presents the properties and design procedure of the configuration matrix of over-actuated marine systems. Performance indices introduced in manipulator robots are extended in over-actuated marine vehicles. Moreover, two novel indices, namely reactive index and robust index, are proposed for configuration matrix design process. The problem is formulated as a multi-objective optimization problem. Simulation and preliminary experimental results show the solutions of the design process.
\end{abstract}

Index Terms-Over-actuated systems, underwater robots, performance indices, multi-objective optimization

\section{INTRODUCTION}

Actuation System (AS) is a pivotal part of a robotic system. It is in charge of realizing the desired fore/torque provided by the control system $\left(\mathbf{F}_{B}^{d}\right)$ (see Figure 1a). Actuation sys-

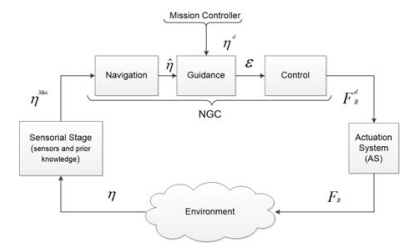

(a) NGC structure augmented with the Actuation System and Sensorial Stage

Fig. 1: Navigation Guidance Control and Actuation System

tem is classically classified into 3 categories: under-actuated, iso-actuated and over-actuated systems, depending on the numbers, positions and directions of thrusters carried by the robot in comparison with the numbers of degrees of freedom (DOFs). However, this classification should be considered along each axis since a robot can be under-actuated with more thrusters than DOFs (for instances, some thrusters have the same direction). The properties of an over-actuated system have been studied in aerospace control, where critical safety is required [1], and for marine vehicles [2] where the harsh oceanic condition may easily produce actuator failures.

The typical structure of an actuation system is shown in Figure $1 \mathrm{~b}$. In this paper, we only focus on the actuator configuration part which is the geometric distribution of actuators. In

This work is supported by Labex NUMEV, Region Occitanie, FEDER, and MUSE linear case, this is called a configuration matrix. The evaluation of the performance of a given geometric configuration of thrusters can be done in evaluating the properties of the configuration matrix with respect to several indices.

Different performance criteria related to the actuators configuration design have been proposed in the literature. For mobile manipulation, manipulability index was proposed in [3]. Attainability was studied using workspace volume estimation in [4], [5], and [6]. Regarding to the comparison of known actuators configurations of over-actuated marine systems, manipulability index, energetic index and force index were proposed in [7]. However, these indices are only used to evaluate a given configuration. Considering reverse process, designing a configuration matrix which optimizes some criteria is not addressed in the literature, especially in the marine field.

This paper focuses on the design process of the configuration matrix of an over-actuated marine system with the performance indices in which some of them, namely manipulability, energetic, workspace indices, are extended from the manipulator robotic field and two of them, namely reactive and robust indices, are originally proposed. The novelties of the paper can be summarized as follows:

1) To extend the performance indices of manipulators to marine systems and to propose two novel indices.

2) To analyze the relationship between different performance indices.

3) To propose a solution for multi-objective formulated optimization problem.

The paper is organized as follows. The used nomenclatures are shown in the section II. Performance indices and problem formulation are depicted in the section III. Mathematical analysis and problem solution are displayed in the next section. Simulation and preliminary experimental results are presented in the section V and VI respectively. Finally, conclusion is given in the section VII. All proofs are given in the appendix section.

\section{NOTATIONS}

This section depicts most of notations used in the paper. However, specific notations will be introduced when needed. In order to illustrate the notations, a given robot configuration is shown in Figure 2a. 


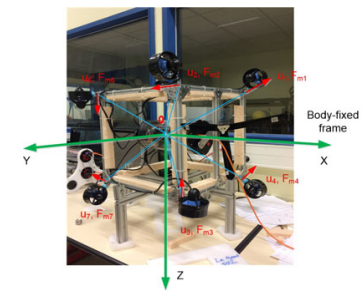

(a) A given robot configuration(CubeRobot) in LIRMM (b) Actuators configuration model

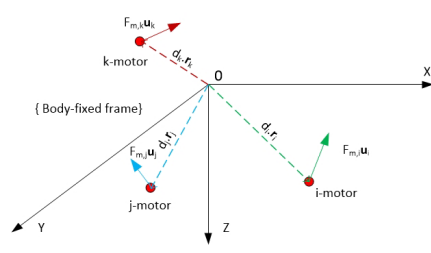

Fig. 2: Configuration model

\begin{tabular}{|c|c|}
\hline $\mathbf{A}$ & Configuration matrix \\
\hline $\mathbf{A}^{+}$ & $\begin{array}{l}\text { Moore-Penrose pseudo-inverse of } \\
\text { A matrix }\end{array}$ \\
\hline $\mathbf{u}_{i}$ & $\begin{array}{l}(3 \times 1) \text { - normalized vector of direc- } \\
\text { tion of the } i^{t h} \text { thruster }\end{array}$ \\
\hline $\mathbf{r}_{i}$ & $\begin{array}{l}(3 \times 1) \text { - normalized vector of posi- } \\
\text { tion of the } i^{t h} \text { thruster }\end{array}$ \\
\hline $\mathbf{F}_{m}$ & $(m \times 1)$ - Force vector of $\mathrm{m}$ thrusters \\
\hline$F_{m, i}$ & Force magnitude of the $i^{t h}$ thruster \\
\hline $\mathbf{F}_{B}^{d}$ & $\begin{array}{l}(6 \times 1) \text { - Desired force (force and } \\
\text { torque) w.r.t body frame }\end{array}$ \\
\hline $\mathbf{F}_{B}=\left(\begin{array}{l}\mathbf{f} \\
\boldsymbol{\tau}\end{array}\right)$ & $\begin{array}{l}(6 \times 1) \text { - Resulting force (force and } \\
\text { torque) w.r.t body frame }\end{array}$ \\
\hline $\mathbf{c}_{m}$ & $(m \times 1)$ - Input vector of thrusters \\
\hline$\otimes$ & Cross product \\
\hline$\|\cdot\|$ & Euclidian norm \\
\hline$\|\cdot\|_{p}$ & p-norm \\
\hline$m$ & the number of thrusters \\
\hline$n$ & $\begin{array}{l}\text { the number of degree of freedoms } \\
\text { (DOFs) }\end{array}$ \\
\hline f & $\begin{array}{l}(3 \times 1) \text {-the vector of force elements } \\
\text { in the resulting force } \mathbf{F}_{B}\end{array}$ \\
\hline$\tau$ & $\begin{array}{l}(3 \times 1) \text {-the vector of torque ele- } \\
\text { ments in the resulting force } \mathbf{F}_{B}\end{array}$ \\
\hline
\end{tabular}

\section{PROBLEM FORMULATION}

\section{A. Model of actuators configuration}

In the configuration matrix design problem, thrusters carried by the robot are characterized by their positions and orientations w.r.t the body frame of the robot. This can be seen in Figures $2 \mathrm{a}$ and $2 \mathrm{~b}$. The configuration matrix $\mathbf{A}$ is described:

$$
\begin{aligned}
\mathbf{A} & =\left(\begin{array}{cccc}
\mathbf{u}_{1} & \mathbf{u}_{2} & \cdots & \mathbf{u}_{m} \\
d_{1} \mathbf{r}_{1} \otimes \mathbf{u}_{1} & d_{2} \mathbf{r}_{2} \otimes \mathbf{u}_{2} & \cdots & d_{m} \mathbf{r}_{m} \otimes \mathbf{u}_{m}
\end{array}\right) \\
& =\left(\begin{array}{llll}
\mathbf{u}_{1} & \mathbf{u}_{2} & \cdots & \mathbf{u}_{m} \\
\boldsymbol{\tau}_{1} & \boldsymbol{\tau}_{2} & \cdots & \boldsymbol{\tau}_{m}
\end{array}\right)=\left(\begin{array}{l}
\mathbf{A}_{1} \\
\mathbf{A}_{2}
\end{array}\right)
\end{aligned}
$$

where $\mathbf{A}_{1}, \mathbf{A}_{2} \in \mathbb{R}^{3 \times m}$ are sub-matrices of $\mathbf{A}$ which concern force and torque elements respectively; $\mathbf{u}_{i}$ and $\mathbf{r}_{i}$ are direction and position vector of the $i^{\text {th }}$ thruster w.r.t the body frame. It is obvious to see that $\boldsymbol{\tau}_{i}^{T} \cdot \mathbf{u}_{i}=0$. This is one of the constraints of the configuration matrix; $m$ is the number of thrusters, $d_{i}$ is the distance from origin of body-frame to the position of $i^{\text {th }}$ thruster.

In this paper, we assume that all distances from thrusters positions to the center of body-frame are the same, $d_{i}=$ const, $i=1 \ldots m$. Without loss of generality, we can assume that $d_{i}=1, i=1, \ldots, m$.

\section{B. Manipulability index}

Manipulability index was first introduced in [8] for manipulator mechanisms. It measures the capability of producing the same force/torque in any direction. It is defined as the condition number of the configuration matrix $\mathbf{A}$ :

$$
I_{m}=\operatorname{Cond}(\mathbf{A})=\frac{\sigma_{\max }}{\sigma_{\min }}
$$

where $\sigma_{\max }$ and $\sigma_{\min }$ are the maximum and minimum singular value of configuration matrix, $\mathbf{A}$, respectively. The objective is to minimize this index. If $I_{m}=1$, the robot is isotropic or if $I_{m}=\infty$ the robot only acts along one direction.

\section{Energetic index}

In fact, energy consumption of a robot depends on many factors such as the mission of the robot, architecture of the robot, and so on. In this paper, energetic index measures the variation of energy consumption of a marine system when the desired force/torque changes. It was first introduced in [7]. However, being different from [7], the norm of thruster force vector, $p_{E}=\left\|\mathbf{F}_{m}\right\|_{2}$, is used to qualify the energy consumption that a marine robot spends to produce forces and torques, and can be calculated as Equation (3).

$$
p_{E}=\left\|\mathbf{F}_{m}\right\|_{2}=\sqrt{\sum_{i=1}^{m} F_{m, i}^{2}}=\left\|\mathbf{A}^{+} . \mathbf{F}_{B}^{d}\right\|_{2}
$$

The energetic index is measured when the normalized vector of desired force and torque change all over a 3D-sphere. Therefore, it is defined as:

$$
I_{e}=\frac{1}{S} \int_{S}\left(w_{e f} p_{E f}+w_{e \tau} p_{E \tau}\right) d S
$$

where $S$ is the surface area of $3 D$ sphere; $w_{e f}$ and $w_{e \tau}$ are weighting coefficients; $p_{E f}, p_{E \tau}$ are derived from $p_{E}$ as:

$\left\{p_{E f}=\left\|\mathbf{A}^{+} \mathbf{F}_{B}^{d}(\mathbf{f})\right\|=\left\|\mathbf{A}^{+}\left(\begin{array}{c}\mathbf{u}_{s} \\ \mathbf{0}\end{array}\right)\right\|, \quad\right.$ for force sphere case $\left\{p_{E \tau}=\left\|\mathbf{A}^{+} \mathbf{F}_{B}^{d}(\boldsymbol{\tau})\right\|=\left\|\mathbf{A}^{+}\left(\begin{array}{c}\mathbf{0} \\ \mathbf{u}_{s}\end{array}\right)\right\|, \quad\right.$ for torque sphere case

where $\mathbf{u}_{s}=\left[\begin{array}{lll}\cos \theta \cos \psi & \sin \theta \cos \psi & \sin \psi\end{array}\right]^{T}$ is a normalized vector in spherical coordinates with $\theta \in[-\pi, \pi]$, and $\psi \in[-\pi / 2, \pi / 2]$.

\section{Workspace index}

Workspace index measures the volume of attainable region of resulting force/torque space w.r.t body frame. It is defined as:

$$
I_{w}=\omega_{w f} \operatorname{Vol}\left(\mathbb{F}_{F}\right)+\omega_{w \tau} \operatorname{Vol}\left(\mathbb{F}_{T}\right)
$$

where $V o l$ is the attainable volume of a space; $\omega_{w f}$ and $\omega_{w \tau}$ are weighting coefficients; $\mathbb{F}_{F}$ and $\mathbb{F}_{T}$ are resulting force and torque space with respect to saturation values of each thruster respectively. 


\section{E. Reactive index}

Reactive index quantifies how fast the actuation system is able to change the orientation of the resulting force $\mathbf{F}_{B}$ (ideally $\mathbf{F}_{B}^{d}$ ). Suppose that the robot is travelling in a direction with a set of thrusters forces $\mathbf{F}_{m 1}$ induced from desired force vector $\mathbf{F}_{B 1}^{d}$. The desired body-frame action changes to another direction (or the same direction with the different manigtude) with the desired force vector $\mathbf{F}_{B 2}^{d}$, so thrusters have to produce another set of thruster forces $\mathbf{F}_{m 2}$. The 2-norm of deviation of thruster forces, $\triangle \mathbf{F}_{m}=\mathbf{F}_{m 1}-\mathbf{F}_{m 2}=\left[\triangle F_{m 1} \triangle F_{m 2} \ldots\right.$ $\left.\triangle F_{m m}\right]^{T}$, is considered as the reactive capability of the robot. Referring to the approximation of characteristic of thrusters as Fig $3 \mathrm{a}$, the response time from $F_{m 1}$ to $F_{m 2}$ is less than the response time from $F_{m 1}$ to $F_{m 3}$ (in linear section, the deadzone of thrusters charactersistics is neglected in this paper). Hence, we have:

$$
\begin{aligned}
& \triangle \mathbf{F}_{m}=\mathbf{A}^{+}\left(\mathbf{F}_{B 1}^{d}-\mathbf{F}_{B 2}^{d}\right)=\mathbf{A}^{+} \triangle \mathbf{F}_{B}^{d} \\
& \left\|\triangle \mathbf{F}_{m}\right\|=\left\|\mathbf{A}^{+} \triangle \mathbf{F}_{B}^{d}\right\| \leq\left\|\mathbf{A}^{+}\right\|\left\|\triangle \mathbf{F}_{B}^{d}\right\| \\
& \frac{\left\|\triangle \mathbf{F}_{m}\right\|}{\left\|\triangle \mathbf{F}_{B}^{d}\right\|} \leq\left\|\mathbf{A}^{+}\right\|
\end{aligned}
$$

From Equation (9), the sensitivity of the thruster forces with respect to desired forces, in other words the variation of thruster forces w.r.t desired forces, is upper-bounded by the norm of pseudo-inverse of the configuration matrix, $\left\|\mathbf{A}^{+}\right\|$. We define the reactive index as:

$$
I_{r e}=\left\|\mathbf{A}^{+}\right\|
$$

It is obvious to see that if this index is small, the robot is more reactive. Then, the objective of design process is to minimize reactive index.


(a) Model of thruster character- (b) Real thruster characteristic(Blue Robotics) istic

Fig. 3: Thruster characteristic

\section{F. Robust index}

This criterion measures the robust level the AS of a marine robot. It means that if any thrusters of the robot fails, the remaining ones can still perform the robot's mission. In particular, for any $\mathbf{F}_{B}^{d}$ vector, there always exists a $\mathbf{F}_{m}$ vector to satisfy the equation $\mathbf{F}_{B}=\mathbf{A} \mathbf{F}_{m}$ and $\mathbf{F}_{B}$ is as close as possible to $\mathbf{F}_{B}^{d}$.
We have:

$$
\mathbf{F}_{B}=\mathbf{A} \mathbf{F}_{m}=\sum_{i=1}^{m} \mathbf{a}_{i} F_{m, i}
$$

where $\mathbf{a}_{i}$ is the $i^{t h}$ column of the matrix $\mathbf{A}$, and $F_{m, i}$ is the force magnitude of $i^{\text {th }}$ thruster.

When one or more thrusters completely fail, the value of $F_{m, i}=0$. Note that in the case where the $i^{t h}$ thruster is partly failed, the value of $F_{m, i}$ can be bounded to a small value (not addressed in this paper). This is equivalent to consider that the corresponding column $\mathbf{a}_{i}$ of the configuration matrix $\mathbf{A}$ equals to zero vector. Therefore, Equation (11) can be written as:

$$
\mathbf{F}_{B}=\mathbf{A}^{\prime} \mathbf{F}_{m}
$$

where $\mathbf{A}^{\prime}$ matrix is the $\mathbf{A}$ matrix with one or more corresponding columns equal zero vectors.

We discuss hereafter the two questions: conditions of the matrix $\mathbf{A}^{\prime}$ to guarantee that the desired action $\left(\mathbf{F}_{B}^{d}\right)$ can still be attainable, and what is the maximum number of thrusters failure?

For addressing these two questions, suppose that $k$-thrusters fail, and the Equation (12) results in 6 equations (dimension of $\mathbf{F}_{B}$ is $\left.6 \times 1\right)$ and $(m-k)$ variables because the matrix $\mathbf{A}^{\prime}$ is $6 \times m$ with $k$ columns are zero vectors. It is obvious to see that if $\operatorname{rank}\left(\mathbf{A}^{\prime}\right)=6$, for given $\mathbf{F}_{B}^{d}$, there always exits $\mathbf{F}_{m}$ such that $\mathbf{F}_{B}=\mathbf{A}^{\prime} \mathbf{F}_{m}$. This can be interpreted as $m-k \geq 6$ or $k \leq m-6$. The conditions of the configuration matrix and the maximum number of thrusters failure that guarantee the robustness of a marine robot are stated as:

1) The maximum of thrusters failure: $m-6$

2) Robust condition: the rank of configuration matrix always equals to 6 , i.e, $\operatorname{rank}\left(\mathbf{A}^{\prime}\right)=6$, if any columns, from 1 to maximum $(m-6)$, of $\mathbf{A}$ matrix equal to zero vectors. If $\operatorname{rank}\left(\mathbf{A}^{\prime}\right)<6$, the system becomes under-actuated, the guidance and control have to change to guarantee the robot's mission. This problem is not addressed in this paper.

We define the robust index as:

$$
I_{\text {ro }}=\operatorname{rank}\left(\left.\mathbf{A}\right|_{\leq m-6}\right)=6
$$

where $\left.\mathbf{A}\right|_{\leq m-6}$ is the $\mathbf{A}$ matrix with the maximum number of columns being zero is $(m-6)$. This novel index will be verified in the solving process of the problem.

\section{G. Configuration matrix design problem}

The design problem is written as:

$$
\begin{array}{cl}
\min _{\mathbf{A}} \mathbf{V}(\mathbf{A})=\min _{\mathbf{A}}\left[I_{m} I_{e} \frac{1}{I_{w}} I_{r e}\right]^{T} \\
\text { s.t } \quad\left\|\mathbf{u}_{i}\right\|=1, i=1,2, \ldots m \\
\left\|\boldsymbol{\tau}_{i}\right\| \leq 1, i=1,2, \ldots m \\
\boldsymbol{\tau}_{i}^{T} \mathbf{u}_{i}=0, i=1,2, \ldots m \\
I_{r o}=\operatorname{rank}\left(\left.\mathbf{A}\right|_{\leq m-6}\right)=n=6
\end{array}
$$

A multi-objective optimization technique, goal attainment approach, is used to find a Pareto solution of the design problem. 


\section{PRoblem SOLUtion}

Our objective is to find an optimal distribution (positions and orientations) of thrusters of the marine system. This can be derived from an optimal configuration matrix $\mathbf{A}$ which is a solution of (14).

\section{A. Mathematical analysis}

The configuration matrix $\mathbf{A}$ has the form as:

$$
\mathbf{A}=\left(\begin{array}{llll}
\mathbf{u}_{1} & \mathbf{u}_{2} & \cdots & \mathbf{u}_{m} \\
\boldsymbol{\tau}_{1} & \boldsymbol{\tau}_{2} & \cdots & \boldsymbol{\tau}_{m}
\end{array}\right)
$$

We have:

$$
\mathbf{B}=\mathbf{A}^{T} \mathbf{A}=\left(\begin{array}{cccc}
\mathbf{u}_{1} & \mathbf{u}_{2} & \cdots & \mathbf{u}_{m} \\
\boldsymbol{\tau}_{1} & \boldsymbol{\tau}_{2} & \cdots & \boldsymbol{\tau}_{m}
\end{array}\right)^{T}\left(\begin{array}{cccc}
\mathbf{u}_{1} & \mathbf{u}_{2} & \cdots & \mathbf{u}_{m} \\
\boldsymbol{\tau}_{1} & \boldsymbol{\tau}_{2} & \cdots & \boldsymbol{\tau}_{m}
\end{array}\right)
$$

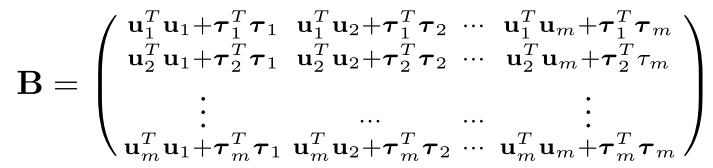

$\mathbf{B}$ is a $m \times m$ symetric matrix where each element is denoted as $b_{i j}$. We have:

$$
\begin{aligned}
\operatorname{Tr}(\mathbf{B}) & =\sum_{i=1}^{m} b_{i i} \\
& =\sum_{i=1}^{m} \lambda_{i}
\end{aligned}
$$

where $\lambda_{i}$ is the $i^{\text {th }}$ eigenvalue of matrix $\mathbf{B}$, and $\operatorname{Tr}(\mathbf{B})$ denotes the trace of matrix $\mathbf{B}$.

From Equations (17), and (18), we have:

$$
\begin{aligned}
\sum_{i=1}^{m} \lambda_{i} & =\sum_{i=1}^{m} \mathbf{u}_{i}^{T} \mathbf{u}_{i}+\boldsymbol{\tau}_{i}^{T} \boldsymbol{\tau}_{i} \\
& =\sum_{i=1}^{m}\left\|\mathbf{u}_{i}\right\|^{2}+\left\|\boldsymbol{\tau}_{i}\right\|^{2} \\
\sum_{i=1}^{m} \lambda_{i} & =\sum_{i=1}^{m}\left(1+\left\|\boldsymbol{\tau}_{i}\right\|^{2}\right)
\end{aligned}
$$

In the case of manipulability index optimization, the condition of configuration matrix $\mathbf{A}$ is $1, \operatorname{cond}(\mathbf{A})=1$. This means that the maximum singular value equals the minimum singular value, $\sigma_{\max }=\sigma_{\min }$. Note that the matrix $\mathbf{A}$ is the $n \times m$ matrix with $n<m$. The matrix $\mathbf{A}$ has $n$ non-zero singular values (we have to guarantee that $\operatorname{rank}(\mathbf{A})=n$ ), then the matrix $\mathbf{B}$ has $n$ non-zero eigenvalues and $m-n$ zero eigenvalues.

In the optimization case of manipulability index, $\operatorname{cond}(\mathbf{A})=1 \Rightarrow \sigma_{\max }=\sigma_{\min }$, we have $\lambda_{i}=\lambda_{\max }=\lambda_{\min }=\lambda(\sigma=\sqrt{\lambda})$. Equation (19) is rewritten:

$$
\begin{aligned}
n \lambda & =m+\sum_{i=1}^{m}\left\|\boldsymbol{\tau}_{i}\right\|^{2} \\
\lambda & =\frac{m}{n}+\frac{1}{n} \sum_{i=1}^{m}\left\|\boldsymbol{\tau}_{i}\right\|^{2}
\end{aligned}
$$

The fact that $\left\|\boldsymbol{\tau}_{i}\right\|^{2} \leq 1$, we have:

$$
\lambda \leq 2 \cdot \frac{m}{n}
$$

Therefore, we have $\lambda_{\max }=2 \frac{m}{n}$ when $\left\|\tau_{i}\right\|^{2}=1$.

In the singular value decomposition of a matrix, when $\operatorname{cond}(\mathbf{A})=1$, the matrix $\mathbf{A}$ can be written as:

$$
\mathbf{A}=\mathbf{U S V}^{T}=\mathbf{U}[\sigma]_{n \times m} \mathbf{V}^{T}
$$

where $\mathbf{U} \in \mathbb{R}^{n \times n}, \mathbf{V} \in \mathbb{R}^{m \times m}$ are orthogonal matrices, $\mathbf{S}=$ $[\sigma]_{n \times m}=\left(\begin{array}{cccc}\sigma & 0 & \cdots & 0 \\ \vdots & \sigma & \cdots & 0 \\ 0 & \cdots & \sigma & 0\end{array}\right) \in \mathbb{R}^{n \times m}$

The pseudo-inverse of matrix $\mathbf{A}$ is $\mathbf{A}^{+}$can be written:

$$
\mathbf{A}^{+}=\mathbf{V S}^{+} \mathbf{U}^{T}=\mathbf{V}\left[\frac{1}{\sigma}\right]_{m \times n} \mathbf{U}^{T}
$$

Where $\mathbf{S}^{+}=\left[\frac{1}{\sigma}\right]_{m \times n}=\left(\begin{array}{ccc}\frac{1}{\sigma} & \cdots & 0 \\ \vdots & \frac{1}{\sigma} & 0 \\ 0 & 0 & \frac{1}{\sigma} \\ 0 & \cdots & 0\end{array}\right) \in \mathbb{R}^{m \times n}$

Our objective with reactive index is to minimize the $\left\|\mathbf{A}^{+}\right\|$. From Equation (23), the reactive index $I_{r e}=\left\|\mathbf{A}^{+}\right\|=\frac{1}{\sigma}$, the minimum value of reactive index is equivalent with the maximum value of $\sigma$. Equality of Equation (21) holds.

In order to minimize the reactive index and manipulability index, the configuration matrix $\mathbf{A}$ is written as the following structure:

$$
\begin{aligned}
\mathbf{A} & =\mathbf{U S V}^{T} \\
& =\mathbf{U}\left(\begin{array}{cccccc}
\sigma & 0 & \cdots & 0 & 0 & 0 \\
0 & \sigma & 0 & \cdots & 0 & 0 \\
0 & 0 & \sigma & 0 & \cdots & 0 \\
\vdots & \vdots & \vdots & \vdots & \vdots & \vdots \\
0 & 0 & 0 & \sigma & 0 & 0
\end{array}\right) \mathbf{V}^{T}
\end{aligned}
$$

where $\mathbf{S}(n \times m)$ is like-diagonal and $\sigma=\sqrt{\lambda}=\sqrt{2 \frac{m}{n}}$; $\mathbf{U}(n \times n)$ and $\mathbf{V}(m \times m)$ are orthogonal matrices ( $\mathbf{U U}^{T}=$ $\left.\mathbf{I}, \mathbf{V V}^{T}=\mathbf{I}\right)$. This results can be used as initial value of numerical optimization process and useful for solving the problem. We continue discussing about the energetic index. First, we introduce a proposition as follows:

Proposition 4.1: Let $\mathbf{M}$ be a $p \times q$ matrix $(p \geq q), \mathbf{M} \in$ $\mathbb{R}^{p \times q}$. For all $\mathbf{x} \in \mathbb{R}^{q}$, if $\mathbf{M}=\mathbf{P} \mathbf{\Sigma} \mathbf{Q}^{T}$, where $\mathbf{P} \in \mathbb{R}^{p \times p}, \mathbf{Q} \in$ 
$\mathbb{R}^{q \times q}$ are orthogonal matrices, $\boldsymbol{\Sigma}=\left(\begin{array}{cccc}\mu & 0 & \cdots & 0 \\ 0 & \mu & \cdots & 0 \\ 0 & \cdots & \mu & 0 \\ 0 & \cdots & 0 & \mu \\ \vdots & \vdots & \vdots & \vdots \\ 0 & 0 & 0 & 0\end{array}\right) \in$

$\mathbb{R}^{p \times q}$, then $\|\mathbf{M x}\|=\|\mathbf{M}\|\|\mathbf{x}\|$.

The proof is given in the appendix.

The energetic index is stated as:

$$
I_{e}=\frac{1}{S} \int_{S}\left(w_{e f}\left\|\mathbf{A}^{+} \mathbf{F}_{B}^{d}(\mathbf{f})\right\|+w_{e \tau}\left\|\mathbf{A}^{+} \mathbf{F}_{B}^{d}(\boldsymbol{\tau})\right\|\right) d S
$$

Choose $w_{e f}=w_{e \tau}=1$ (because desired force vectors, $\mathbf{F}_{B}^{d}(\mathbf{f}), \mathbf{F}_{B}^{d}(\boldsymbol{\tau})$, are normalized), we have:

$$
I_{e}=\frac{1}{S} \int_{S}\left(\left\|\mathbf{A}^{+} \mathbf{F}_{B}^{d}(\mathbf{f})\right\|+\left\|\mathbf{A}^{+} \mathbf{F}_{B}^{d}(\boldsymbol{\tau})\right\|\right) d S
$$

In case where a solution minimizes reactive index and manipulability index, the configuration matrix $\mathbf{A}(n \times m)$ has the form as Equation (24), therefore the pseudo-inverse matrix $\mathbf{A}^{+}(m \times n, \quad m>n)$ has the following structure:

$$
\mathbf{A}^{+}=\mathbf{V S}^{+} \mathbf{U}^{T}=\mathbf{V}\left(\begin{array}{cccc}
\frac{1}{\sigma} & 0 & \cdots & 0 \\
0 & \frac{1}{\sigma} & \cdots & 0 \\
0 & \cdots & \frac{1}{\sigma} & 0 \\
0 & \cdots & 0 & \frac{1}{\sigma} \\
\vdots & \vdots & \vdots & \vdots \\
0 & 0 & 0 & 0
\end{array}\right) \mathbf{U}^{T}
$$

where $\mathbf{V}, \mathbf{U}$ are orthogonal matrices.

It is clear that matrix $\mathbf{A}^{+}$satisfies the condition of Proposition 4.1. Applying this proposition, we have: $\left\|\mathbf{A}^{+} \mathbf{F}_{B}^{d}(\mathbf{f})\right\|=$ $\left\|\mathbf{A}^{+}\right\|\left\|\mathbf{F}_{B}^{d}(\mathbf{f})\right\|$ and $\left\|\mathbf{A}^{+} \mathbf{F}_{B}^{d}(\boldsymbol{\tau})\right\|=\left\|\mathbf{A}^{+}\right\|\left\|\mathbf{F}_{B}^{d}(\boldsymbol{\tau})\right\|$. Therefore, Equation (26) becomes:

$$
\begin{aligned}
I_{e} & =\frac{1}{S} \int_{S}\left(\left\|\mathbf{A}^{+}\right\|\left\|\mathbf{F}_{B}^{d}(\mathbf{f})\right\|+\left\|\mathbf{A}^{+}\right\|\left\|\mathbf{F}_{B}^{d}(\boldsymbol{\tau})\right\|\right) d S \\
& =\frac{1}{S}\left\|\mathbf{A}^{+}\right\| \int_{S}\left(\left\|\mathbf{F}_{B}^{d}(\mathbf{f})\right\|+\left\|\mathbf{F}_{B}^{d}(\boldsymbol{\tau})\right\|\right) d S \\
& =2\left\|\mathbf{A}^{+}\right\|
\end{aligned}
$$

For aforementioned mathematical analysis of the energetic index, we can see that the energetic index belongs to the norm of pseudo-inverse of configuration matrix, $I_{\text {re }}=2\left\|\mathbf{A}^{+}\right\|$, when the configuration matrix $\mathbf{A}$ has the form of (24).

We discuss about the upper-bound of workspace index. For units consistency, the workspace index for force space and for torque space are investigate separately, denoted as $I_{w f}$ and $I_{w \tau}$ respectively. Recall that the objective of workspace index is to maximize the volume of resulting force space $\left(\mathbf{F}_{B}\right.$ space) including resulting space for force and resulting space for torque with given thrusters force space $\left(\mathbf{F}_{m}\right.$ space).

The fact that for all vector $\mathbf{F}_{m} \in \mathbb{R}^{m},\left\|\mathbf{A F}_{m}\right\| \leq$ $\|\mathbf{A}\|\left\|\mathbf{F}_{m}\right\|$. The volume of the resulting force space is maximum when the equality holds. Following Figure 4, the volume of resulting force spaces $\left(\mathbf{F}_{B}\right)$ (force and torque spaces) are always less than the volume of exterior hyper-sphere of $\mathbf{F}_{B}$

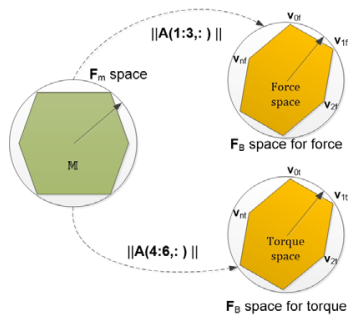

Fig. 4: Upper-bound of resulting force space

spaces of force and torque (may be the circumscribed spheres or not). This means that:

$$
\begin{aligned}
& I_{w F} \leq \text { Volume }(\mathbf{B}(R 1)) \\
& I_{w T} \leq \text { Volume }(\mathbf{B}(R 2))
\end{aligned}
$$

where $\mathbf{B}(R 1)$ and $\mathbf{B}(R 2)$ are an Euclidean balls of radius $R 1=\|\mathbf{A}(\mathbf{1}: \mathbf{3},:)\|\left\|\mathbf{F}_{m}\right\|=\left\|\mathbf{A}_{1}\right\|\left\|\mathbf{F}_{m}\right\|$ and $R 2=$ $\|\mathbf{A}(\mathbf{4}: \mathbf{6},:)\|\left\|\mathbf{F}_{m}\right\|=\left\|\mathbf{A}_{2}\right\|\left\|\mathbf{F}_{m}\right\|$ respectively; $\mathbf{A}(\mathbf{1}: \mathbf{3},:)$ is the $\mathbf{A}$ matrix with three first rows, and $\mathbf{A}(\mathbf{4}: \mathbf{6},:)$ is the $\mathbf{A}$ matrix with three last rows.

The fact that $n$-dimensional volume of an Euclidean ball of radius $R$ in $n$-dimensional Euclidean space is:

$$
V_{n}(R)= \begin{cases}\frac{\pi^{k}}{k !} R^{2 k}, & \text { if } n=2 k \\ \frac{2^{k+1} \pi^{k}}{(2 k+1) ! !} R^{2 k+1}, & \text { if } n=2 k+1\end{cases}
$$

where $(2 k+1) ! !=1.3 .5 \ldots(2 k-1) \cdot(2 k+1)$.

Proposition 4.2: If the configuration matrix $\mathbf{A}$ has the form of (24) then $\operatorname{cond}\left(\mathbf{A}_{1}\right)=\operatorname{cond}\left(\mathbf{A}_{2}\right)=1$ and $\left\|\mathbf{A}_{1}\right\|=$ $\left\|\mathbf{A}_{2}\right\|=\sigma$

The proof is given in the appendix.

From (29) and (30) and Proposition 4.2, it is obvious to get the upper-bound of resulting spaces of force and torque of the system, and then the upper-bound of workspace index. Normally, the weighting coefficients in workspace index are chosen as 1 because of our assumption for $d_{i}$.

\section{B. Problem solution}

The multi-objective optimization problem (14) with aforementioned analyses derives a choice of a solving method, called goal attainment approach. The underlying idea of this method is to minimize the deviation of desired values and guessing values. Our problem using goal attainment method becomes:

$$
\begin{aligned}
\min _{\mathbf{A}, \gamma} \gamma & \\
\text { s.t } & \mathbf{A} \in \overline{\mathbb{A}} \\
& \mathbf{V}(\mathbf{A})-\mathbf{w} \gamma \leq \mathbf{V}_{\text {goal }}
\end{aligned}
$$

where $\overline{\mathbb{A}}=\mathbb{A} \backslash I_{\text {ro }}$, i.e, A set without robust index $I_{\text {ro }}, \gamma$ is a slack vector variable, $\mathbf{V}_{\text {goal }}=\left[\begin{array}{llll}I_{m}^{d} & I_{e}^{d} & \frac{1}{I^{d}} & I_{r e}^{d}\end{array}\right]$ is the desired objective vector, $\mathbf{w}$ is a weighting vector which can be chosen by Decision Maker. The goal attainment method with two objective functions is illustrated in Figure 5. By altering w vector, we get Pareto optimal solutions. Therefore, the problem 


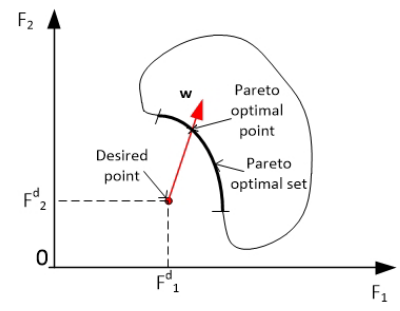

Fig. 5: Goal attainment method with two objective functions

solving process includes two phase:

1) Phase 1: Find one Pareto solution of configuration matrix with goal attainment method.

2) Phase 2: Check robust index of the chosen solution in phase 1.

The optimization toolbox in Matlab environment is used to solve and simulate our problem.

\section{Simulation Results}

This section shows the results with $m=8$ thrusters and $n=6$ DOFs: the general case, in which positions and orientations of thrusters are unknown, and the given position case, in which the positions of thrusters are given. After that, the comparison between two configurations is shown to prove the aforementioned approach.

\section{A. General case}

Solving the problem (14) for the general case, one Pareto optimal configuration matrix is found as:

$\mathbf{A}=\left(\begin{array}{cccccccc}-0.8891 & -0.3645 & 0.5438 & 0.9879 & 0.3134 & 0.0148 & 0.0495 & 0.6090 \\ -0.0985 & -0.3036 & -0.5911 & -0.0608 & -0.9493 & 0.0515 & 0.8919 & 0.7158 \\ 0.4471 & 0.8803 & 0.5957 & 0.1429 & 0.0260 & 0.9986 & 0.4495 & 0.3417 \\ -0.4308 & 0.4701 & -0.8386 & 0.0379 & -0.1336 & 0.5628 & -0.9972 & 0.4758 \\ 0.5107 & 0.7561 & -0.4103 & 0.9868 & -0.0712 & -0.8259 & 0.0690 & 0.0149 \\ -0.7441 & 0.4554 & 0.3583 & 0.1577 & -0.9885 & 0.0342 & -0.0272 & -0.8794\end{array}\right)$

The positions and orientations of thrusters are shown in Figure $6 \mathrm{a}$, the attainable force space and torque space are illustrated in Figures $6 \mathrm{~b}$ and $6 \mathrm{c}$, respectively. It is easy to see that these spaces are almost isotropic.

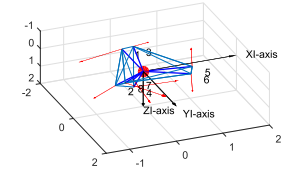

(a) Robot design

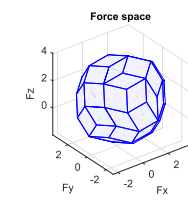

(b) Attainable force space

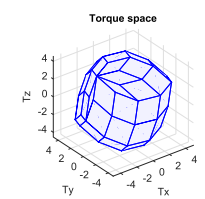

(c) Attainable torque space

Fig. 6: General case simulation

\section{B. Given position case}

Solving the problem (14) with given position of thrusters (a cube shape with the motors installed on the 8 cube corners), one optimal configuration matrix is derived as:

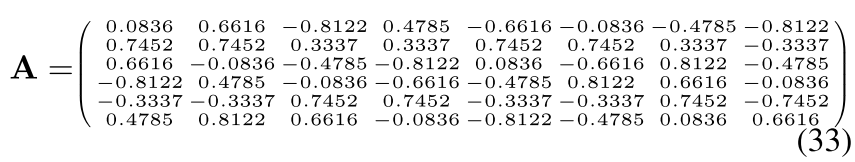

Figure $7 \mathrm{a}$ shows the positions and directions of thrusters of robots. Figures $7 b$ and $7 c$ show the attainable force and torque space respectively. From these figures, it is obvious to see that these spaces are also almost isotropic.

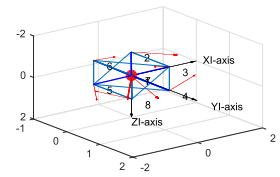

(a) Robot design

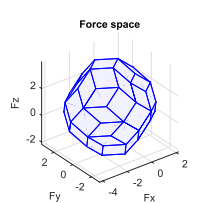

(b) Attainable force space

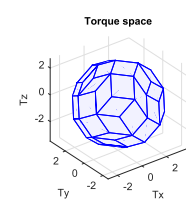

(c) Attainable torque space

Fig. 7: Given position case simulation

\section{A comparison of two cases}

In this section, a comparison of two configurations is illustrated. The first one is a normal configuration $\left(\mathbf{C}^{1}\right)$ in which the thrusters are distributed vertically or horizontally(in practice, this configuration is easier to install as Figure 2a). The configuration matrix of $\mathbf{C}^{1}$ configuration, denoted $\mathbf{A}_{1}$, is shown in Equation (34).

$$
\mathbf{A}_{1}=\left(\begin{array}{cccccccc}
0 & 1 & 0 & 0 & 0 & 0 & -1 & 0 \\
1 & 0 & 0 & -1 & 1 & 0 & 0 & 0 \\
0 & 0 & -1 & 0 & 0 & 1 & 0 & -1 \\
0.27 & 0 & -0.27 & 0.27 & 0.27 & 0.27 & 0 & 0.27 \\
0 & -0.27 & 0.27 & 0 & 0 & 0.27 & -0.27 & -0.27 \\
0.27 & -0.27 & 0 & -0.27 & -0.27 & 0 & 0.27 & 0
\end{array}\right)
$$

The second one $\left(\mathbf{C}^{2}\right)$ is an optimal configuration, denoted as $\mathbf{A}_{2}$, which is a solution of optimization problem (given position case) and the optimal configuration matrix is shown in Equation (35).

$\mathbf{A}_{2}=\left(\begin{array}{cccccccc}0.6616 & -0.8122 & 0.4785 & 0.0836 & -0.0836 & -0.4785 & -0.8122 & -0.6616 \\ 0.7452 & 0.3337 & 0.3337 & 0.7452 & 0.7452 & 0.3337 & -0.3337 & 0.7452 \\ -0.0836 & -0.4785 & -0.8122 & 0.6616 & -0.6616 & 0.8122 & -0.4785 & 0.0836 \\ 0.1608 & 0.0111 & -0.2459 & -0.3708 & 0.3642 & 0.2015 & 0.0011 & -0.1658 \\ -0.0989 & 0.3556 & 0.3633 & -0.0989 & -0.1056 & 0.3508 & -0.3456 & -0.1056 \\ 0.3906 & 0.2292 & 0.0044 & 0.1583 & -0.1649 & -0.0254 & 0.2392 & -0.3708\end{array}\right)$

Note that the configuration matrices $\mathbf{A}_{1}$ and $\mathbf{A}_{2}$ are calibrated with corresponding geometrical properties of real cube robot in LIRMM. The attainable force space and torque space corresponding with two configurations $\mathbf{C}^{1}$ and $\mathbf{C}^{2}$ are illustrated in Figures $8 \mathrm{a}$ and $8 \mathrm{~b}$. It is obvious to see that the $\mathbf{C}^{2}$ configuration is more isotropic than the $\mathbf{C}^{1}$ configuration. 


\begin{tabular}{|c|c|c|c|}
\hline No. & Indices & $\mathbf{C}^{1}$ & $\mathbf{C}^{2}$ \\
\hline 1 & $I_{m}$ & 4.7729 & 2.5592 \\
\hline 2 & $I_{e}$ & 5.4387 & 3.1243 \\
\hline 3 & $I_{w}$ & $6.6466 \mathrm{e}+06$ & $1.0919 \mathrm{e}+07$ \\
\hline 4 & $I_{r e}$ & 2.7194 & 1.5622 \\
\hline 5 & $I_{r o}$ & false & true \\
\hline
\end{tabular}

TABLE I: Comparison between two configurations

However, for some specific points of attainable fore and torque space, the $\mathbf{C}^{1}$ configuration is larger than the $\mathbf{C}^{2}$ configuration.

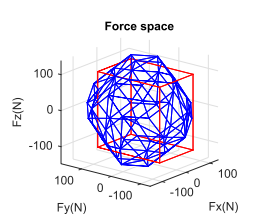

(a) $\mathbf{C}^{1}$ (red), $\mathbf{C}^{2}$ (blue)

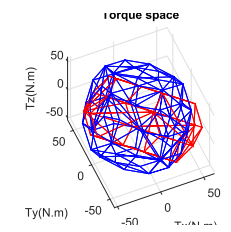

(b) $\mathbf{C}^{1}$ (red), $\mathbf{C}^{2}$ (blue)
Fig. 8: Attainable spaces for different configurations

Thanks to the properties of matrices $\mathbf{A}_{1}$ and $\mathbf{A}_{2}$ (Equation (34) and (35)) and the motor characteristic (3b), Table I shows the values of performance indices for two configurations. The performances of $\mathbf{C}^{2}$ configuration are better than ones of $\mathbf{C}^{1}$. Because of the calibration (the distance $d_{i}$ is different between motors), the manipulability index $\left(I_{m}\right)$ is larger than 1 .

In order to verify the attainability of two configurations (workspace index), incremental torques are applied about X, Y, and $\mathrm{Z}$ axis respectively (Figures 9a, 10a, and 11a), the corresponding PWM (Pulse Width Modulation) inputs of 8 thrusters are computed. The results are shown in Figures $9 b, 9 c, 10 b$, $10 \mathrm{c}, 11 \mathrm{~b}$, and $11 \mathrm{c}$ in which the two PWM's saturation values of thrusters (upper saturation value: 1900, lower saturation value: 1100) are plotted with two bold lines. We can see that the performances of the robot with two configurations are almost the same with the rotation about $\mathrm{X}$ and $\mathrm{Y}$ axis. However, the $\mathbf{C}^{2}$ configuration shows better performance with the rotation about Z-axis. In fact, the thrusters with $\mathbf{C}^{1}$ configuration reach saturations very earlier in comparison with the thrusters with $\mathrm{C}^{2}$ configuration (Figures $11 \mathrm{~b}$ and $11 \mathrm{c}$ ).

In order to validate the robustness of the optimal configuration $\left(\mathbf{C}^{2}\right)$ in comparison with the normal configuration $\left(\mathbf{C}^{1}\right)$, the rank of matrices $\mathbf{A}_{1}$ and $\mathbf{A}_{2}$ is checked when arbitrary one or two columns have been nullified. When the resulting matrices are rank deficient, this means that the robustness is not guaranteed because one DOF is not actuated. Therefore, we can not control all 6 DOFs independently. The robust index in Table I shows the checking results. In particular, when the $5^{\text {th }}$ thruster of $\mathbf{C}^{1}$ configuration fails, the robustness is not guaranteed.

\section{PRELIMINARY EXPERIMENTAL RESUlts}

This section presents some preliminary experimental studies to compare normal configuration, $\mathbf{C}^{1}$, and optimal configura-

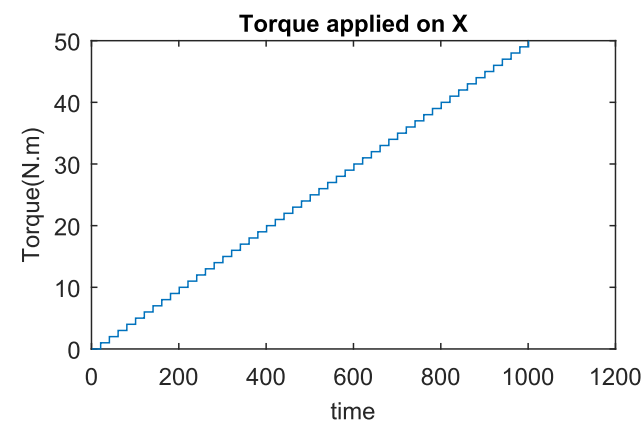

(a) Applied torque about $\mathrm{X}$-axis



(b) PWM inputs of $\mathrm{C} 1$

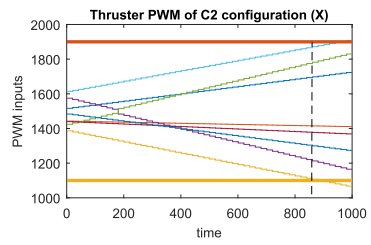

(c) PWM inputs of $\mathrm{C} 2$
Fig. 9: The simulation of cube rotation about $\mathrm{X}$-axis for $\mathbf{C}^{1}$ and $\mathbf{C}^{2}$

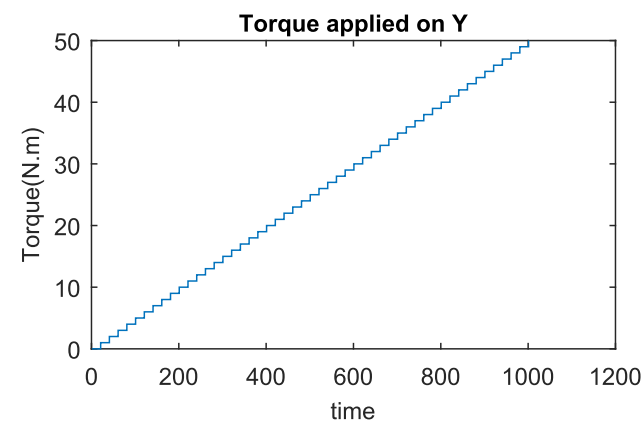

(a) Applied torque about Y-axis



(b) PWM inputs of $\mathrm{C} 1$

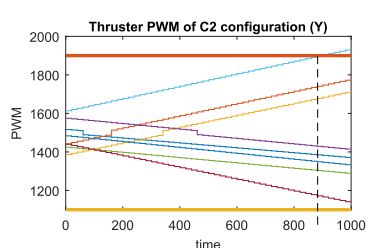

(c) PWM inputs of $\mathrm{C} 2$
Fig. 10: The simulation of cube rotation about $\mathrm{Y}$-axis for $\mathbf{C}^{1}$ and $\mathbf{C}^{2}$

tion, $\mathrm{C}^{2}$, of the cube robot. An incremental torques about Xaxis, Y-axis, and Z-axis are applied on cube robot respectively, angular velocities and PWM input values are stored for evaluating these two configurations. For safety, the experiment will be stopped when one thruster reaches the saturation values. The experimental results are shown in Figures 12,13 and 14.

For X-axis rotation, the performances of both configurations are almost the same. Nevertheless, for Y-axis rotation, the performance of $\mathbf{C}^{2}$ configuration is better than one of $\mathbf{C}^{1}$ 


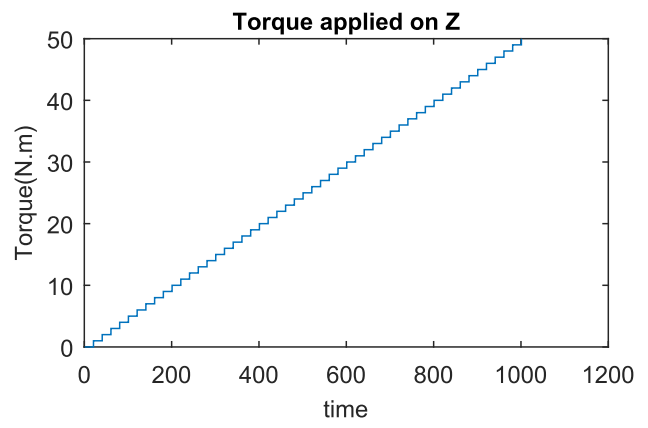

(a) Applied torque about $\mathrm{Z}$-axis

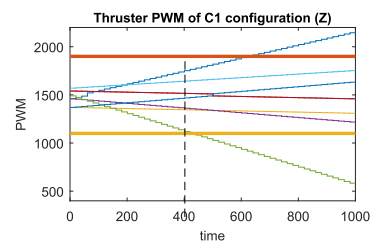

(b) PWM inputs of $\mathrm{C} 1$

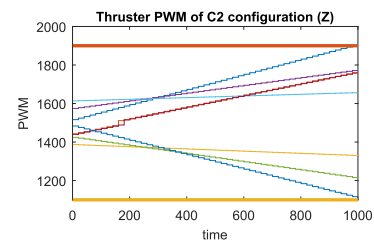

(c) PWM inputs of $\mathrm{C} 2$
Fig. 11: The simulation of cube rotation about $\mathrm{Z}$-axis for $\mathbf{C}^{1}$ and $\mathbf{C}^{2}$

configuration (Figure 13). In fact, for $\mathbf{C}^{1}$ configuration, the one of thrusters get saturation value at time instant $771 \mathrm{~s}$ and the experimentation stops, while the robot continues to operate after that time for $\mathbf{C}^{2}$ configuration. This is clearer for Z-axis rotation experiment (Figure 14). The thrusters of $\mathbf{C}^{1}$ configuration stop quite earlier, at time instant $451 \mathrm{~s}$, in comparison with the thrusters of $\mathbf{C}^{2}$ configuration. Therefore, the attainability (workspace index) of $\mathbf{C}^{2}$ configuration is better than $\mathbf{C}^{1}$ configuration.

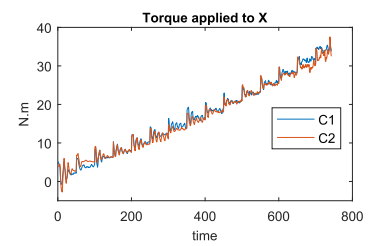

(a) Applied torque about $\mathrm{X}$-axis

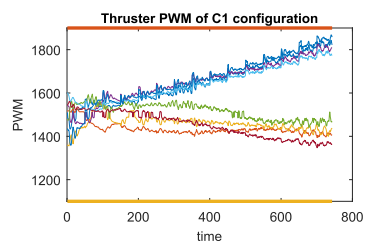

(c) PWM inputs of $\mathrm{C} 1$

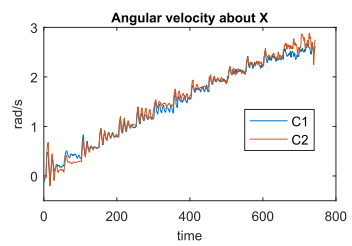

(b) Angular velocities

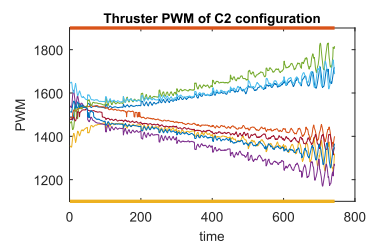

(d) PWM inputs of $\mathrm{C} 2$
Fig. 12: The cube rotates about $\mathrm{X}$-axis for $\mathbf{C}^{1}$ and $\mathbf{C}^{2}$

In the next section, we verify the energy spending during these experiments for two configurations. An energy-like cri-

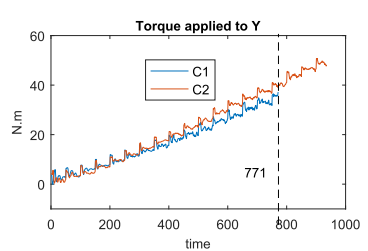

(a) Applied torque about Y-axis

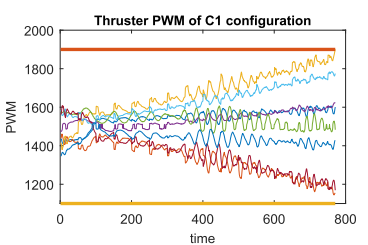

(c) PWM inputs of $\mathrm{C} 1$



(b) Angular velocities

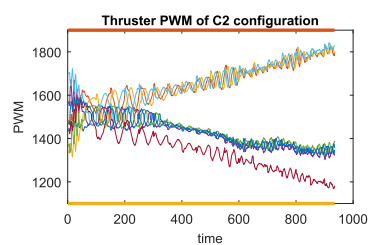

(d) PWM inputs of $\mathrm{C} 2$
Fig. 13: The cube rotates about $\mathrm{Y}$-axis for $\mathbf{C}^{1}$ and $\mathbf{C}^{2}$

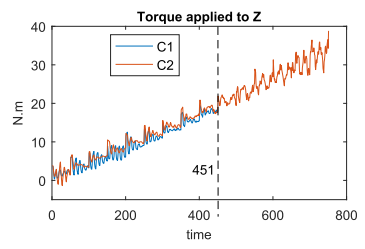

(a) Applied torque about $\mathrm{Z}$-axis

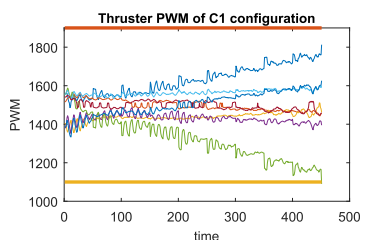

(c) PWM inputs of $\mathrm{C} 1$

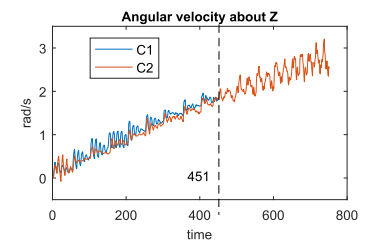

(b) Angular velocities

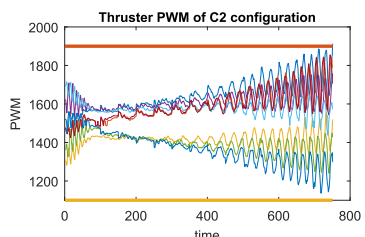

(d) PWM inputs of $\mathrm{C} 2$
Fig. 14: The cube rotates about $\mathbf{Z}$-axis for $\mathbf{C}^{1}$ and $\mathbf{C}^{2}$

terion is proposed:

$$
\mathbf{E}=\sum_{i=1}^{m} \int_{t=0}^{T} P W M^{i}(t) d t
$$

where $m$ is the number of thrusters, $T$ is the time of experiment, $P W M^{i}(t)$ is PWM inputs of $i^{t h}$ thruster.

Table II shows the energy consumption of robot during three rotations experiments. For $\mathrm{X}$-axis rotation, the attainability of two configurations is the same but the the spent energy of $\mathbf{C}^{2}$ configuration is lower. For $\mathrm{Y}$-axis and $\mathrm{Z}$-axis rotation, the duration of experiments of $\mathbf{C}^{2}$ configuration is longer, the energy consumption, therefore, is higher.

\begin{tabular}{|c|c|c|c|}
\hline No. & Rotation & $\mathbf{E}_{\mathbf{C}^{1}}$ & $\mathbf{E}_{\mathbf{C}^{2}}$ \\
\hline 1 & $X$ & $7.2303 \mathrm{e}+04$ & $6.9603 \mathrm{e}+04$ \\
\hline 2 & $Y$ & $7.5480 \mathrm{e}+04$ & $1.0590 \mathrm{e}+05$ \\
\hline 3 & $Z$ & $3.1637 \mathrm{e}+04$ & $7.4350 \mathrm{e}+04$ \\
\hline
\end{tabular}

TABLE II: Energy consumption of two configurations 
Table III shows the comparison of energy consumption of two configurations with the same time duration. For Y-axis rotation, the energy value of $\mathbf{C}^{2}$ configuration is lower than one of $\mathbf{C}^{1}$ configuration. However, for $\mathrm{Z}$-axis, the energy values of $\mathbf{C}^{2}$ configuration is higher. This happens because the robot dived deeper for $\mathbf{C}^{2}$ configuration experiments of $\mathrm{Z}$-axis rotation, the robot had to deliver more power to keep at higher constant depth.

\begin{tabular}{|c|c|c|c|}
\hline No. & Rotation & $\mathbf{E}_{\mathbf{C}^{1}}$ & $\mathbf{E}_{\mathbf{C}^{2}}$ \\
\hline 1 & $Y$ & $7.5480 \mathrm{e}+04$ & $7.2715 \mathrm{e}+04$ \\
\hline 2 & $Z$ & $3.1637 \mathrm{e}+04$ & $3.3312 \mathrm{e}+04$ \\
\hline
\end{tabular}

TABLE III: Energy consumption of two configurations with the same time duration

\section{CONCLUSION}

This paper presents the optimal design of geometric distribution of thrusters of marine systems, mathematically described as a configuration matrix. Three performance indices of manipulators are extended to over-actuated marine systems and two novel indices are proposed. The mathematical analysis of performance indices are also studied. One Pareto solution is found and the simulation and preliminary experimental results shows the effectiveness of the design. For the next researches, finding all Pareto solutions are quite attractive and more experiments are carried out to validate all performance indices. Moreover, dynamic configuration matrix design depending the robot's mission and other issues is still open and interesting topic.

\section{ACKNOWLEDGEMENTS}

The authors thank to Labex NUMEV, Region Occitanie, FEDER, and MUSE for supporting this research, and also express special thanks to Mr Pascal Lepinay and Dr Benoit Ropars for their supports in doing experiments.

\section{APPENDIX}

Proposition A.1: Let $\mathbf{M}$ be a $p \times q$ matrix $(p \geq q), \mathbf{M} \in$ $\mathbb{R}^{p \times q}$. For all $\mathbf{x} \in \mathbb{R}^{q}$, if $\mathbf{M}=\mathbf{P} \boldsymbol{\Sigma} \mathbf{Q}^{T}$, where $\mathbf{P} \in \mathbb{R}^{p \times p}, \mathbf{Q} \in$ $\mathbb{R}^{q \times q}$ are orthogonal matrices, $\boldsymbol{\Sigma}=\left(\begin{array}{cccc}\mu & 0 & \cdots & 0 \\ 0 & \mu & \cdots & 0 \\ 0 & \cdots & \mu & 0 \\ 0 & \cdots & 0 & \mu \\ \vdots & \vdots & \vdots & \vdots \\ 0 & 0 & 0 & 0\end{array}\right) \in$ $\mathbb{R}^{p \times q}$ then $\|\mathbf{M x}\|=\|\mathbf{M}\|\|\mathbf{x}\|$.

Proof We have:

$$
\|\mathbf{M} \mathbf{x}\|^{2}=(\mathbf{M x})^{T}(\mathbf{M x})=\mathbf{x}^{T} \mathbf{M}^{T} \mathbf{M} \mathbf{x}
$$

With $\mathbf{M}=\mathbf{P} \boldsymbol{\Sigma} \mathbf{Q}^{T}$

$$
\begin{aligned}
\|\mathbf{M} \mathbf{x}\|^{2} & =\mathbf{x}^{T}\left(\mathbf{P} \boldsymbol{\Sigma} \mathbf{Q}^{T}\right)^{T}\left(\mathbf{P} \boldsymbol{\Sigma} \mathbf{Q}^{T}\right) \mathbf{x} \\
& =\mathbf{x}^{T} \mathbf{Q} \boldsymbol{\Sigma}^{T} \mathbf{P}^{T} \mathbf{P} \boldsymbol{\Sigma} \mathbf{Q}^{T} \mathbf{x} \\
& =\mathbf{x}^{T} \mathbf{Q} \boldsymbol{\Sigma}^{T} \mathbf{\Sigma} \mathbf{Q}^{T} \mathbf{x}
\end{aligned}
$$

We have:

$$
\begin{aligned}
\boldsymbol{\Sigma}^{T} \boldsymbol{\Sigma} & =\left(\begin{array}{cccc}
\mu & 0 & \cdots & 0 \\
0 & \mu & \cdots & 0 \\
0 & \cdots & \mu & 0 \\
0 & \cdots & 0 & \mu \\
\vdots & \vdots & \vdots & \vdots \\
0 & 0 & 0 & 0
\end{array}\right)\left(\begin{array}{cccc}
\mu & 0 & \cdots & 0 \\
0 & \mu & \cdots & 0 \\
0 & \cdots & \mu & 0 \\
0 & \cdots & 0 & \mu \\
\vdots & \vdots & \vdots & \vdots \\
0 & 0 & 0 & 0
\end{array}\right) \\
& =\left(\begin{array}{cccc}
\mu^{2} & 0 & \cdots & 0 \\
0 & \mu^{2} & \cdots & 0 \\
\vdots & \vdots & \vdots & \vdots \\
0 & \cdots & 0 & \mu^{2}
\end{array}\right)=\mu^{2} \mathbf{I}
\end{aligned}
$$

where $\mathbf{I}$ is $q \times q$ identity matrix.

Replacing the equation (39) to (38), we have:

$$
\begin{aligned}
\|\mathbf{M} \mathbf{x}\|^{2} & =\mathbf{x}^{T} \mathbf{V} \mu^{2} \mathbf{I V}^{T} \mathbf{x} \\
& =\mu^{2} \mathbf{x}^{T} \mathbf{x}=\|\mathbf{M}\|^{2}\|\mathbf{x}\|^{2}
\end{aligned}
$$

Therefore, $\|\mathbf{M x}\|=\|\mathbf{M}\|\|\mathbf{x}\|$.

Proposition A.2: If the configuration matrix $\mathbf{A}$ has the form of (24) then $\operatorname{cond}\left(\mathbf{A}_{1}\right)=\operatorname{cond}\left(\mathbf{A}_{2}\right)=1$ and $\left\|\mathbf{A}_{1}\right\|=$ $\left\|\mathbf{A}_{2}\right\|=\sigma$

Proof We have:

$$
\begin{aligned}
\mathbf{A A}^{T} & =\left(\mathbf{U S V}^{T}\right)\left(\mathbf{U S V}^{T}\right)^{T}=\mathbf{U S V}^{T} \mathbf{V S}^{T} \mathbf{U}^{T} \\
& =\mathbf{U S S}^{T} \mathbf{U}^{T}=\sigma^{2} \mathbf{I}
\end{aligned}
$$

On the other hand:

$$
\begin{aligned}
\mathbf{A A}^{T} & =\left(\begin{array}{l}
\mathbf{A}_{1} \\
\mathbf{A}_{2}
\end{array}\right)\left(\begin{array}{l}
\mathbf{A}_{1} \\
\mathbf{A}_{2}
\end{array}\right)^{T}=\left(\begin{array}{l}
\mathbf{A}_{1} \\
\mathbf{A}_{2}
\end{array}\right)\left(\mathbf{A}_{1}^{T} \mathbf{A}_{2}^{T}\right) \\
& =\left(\begin{array}{l}
\mathbf{A}_{1} \mathbf{A}_{1}^{T} \\
\mathbf{A}_{2} \mathbf{A}_{2}^{T}
\end{array}\right)
\end{aligned}
$$

From (41) and (42), we have:

$$
\begin{aligned}
& \mathbf{A}_{1} \mathbf{A}_{1}^{T}=\sigma^{2} \mathbf{I}_{1} \\
& \mathbf{A}_{2} \mathbf{A}_{2}^{T}=\sigma^{2} \mathbf{I}_{2}
\end{aligned}
$$

where $\mathbf{I}_{1}$ and $\mathbf{I}_{2}$ are partitioned matrices of matrix $\mathbf{I}$.

From (43) and the uniqueness of singular value decomposition [9], it is obvious to get the structures of $\mathbf{A}_{1}$ and $\mathbf{A}_{2}$ are the same as (24) with different dimensions. Therefore, $\operatorname{cond}\left(\mathbf{A}_{1}\right)=\operatorname{cond}\left(\mathbf{A}_{1}\right)=1$ and $\left\|\mathbf{A}_{1}\right\|=\left\|\mathbf{A}_{2}\right\|=\sigma$.

\section{REFERENCES}

[1] W. S. Levine, The Control Systems Handbook: Control System Applications. CRC press, 2010.

[2] B. Ropars, L. Lapierre, A. Lasbouygues, D. Andreu, and R. Zapata, "Redundant actuation system of an underwater vehicle," Ocean Engineering, vol. 151, pp. 276 - 289, 2018. [Online]. Available: http://www.sciencedirect.com/science/article/pii/S0029801817307473

[3] T. Yoshikawa, "Dynamic manipulability of robot manipulators," in Proceedings. 1985 IEEE International Conference on Robotics and Automation, vol. 2, Mar 1985, pp. 1033-1038.

[4] A. Kumar and K. Waldron, "The workspaces of a mechanical manipulator," Journal of Mechanical Design, vol. 103, no. 3, pp. 665-672, 1981.

[5] B. Paden and S. Sastry, "Optimal kinematic design of 6r manipulators," The International Journal of Robotics Research, vol. 7, no. 2, pp. 43-61, 1988. 
[6] F. C. Park and R. W. Brockett, "Kinematic dexterity of robotic mechanisms," The International Journal of Robotics Research, vol. 13, no. 1, pp. 1-15, 1994.

[7] F. Pierrot, M. Benoit, and P. Dauchez, "Optimal thruster configuration for omni-directional underwater vehicles. samos: a pythagorean solution," in OCEANS '98 Conference Proceedings, vol. 2, Sep 1998, pp. 655-659 vol.2.

[8] T. Yoshikawa, "Manipulability of robotic mechanisms," The international journal of Robotics Research, vol. 4, no. 2, pp. 3-9, 1985.

[9] L. N. Trefethen and D. Bau III, Numerical linear algebra. Siam, 1997, vol. 50 . 